\title{
GENERIC LIGHTLIKE SUBMANIFOLDS OF AN INDEFINITE KAEHLER MANIFOLD WITH A SEMI-SYMMETRIC NON-METRIC CONNECTION
}

\begin{abstract}
Recently, this author studied lightlike hypersurfaces of an indefinite Kaehler manifold endowed with a semi-symmetric non-metric connection in [7]. Further we study this subject. The object of study in this paper is generic lightlike submanifolds of an indefinite Kaehler manifold endowed with a semi-symmetric nonmetric connection such that the induced structure tensor field on the submanifolds is recurrent or Lie recurrent.
\end{abstract}

Key words: generic lightlike submanifold, semi-symmetric nonmetric connection, indefinite Kaehler manifold

2010 Mathematical Subject Classification: 53C25, 53C40, $53 C 50$

1. Introduction. A lightlike submanifold $M$ of an indefinite almost complex manifold $\bar{M}$, with an indefinite almost complex structure $J$, is called generic lightlike submanifold if there exists a screen distribution $S(T M)$ of $M$ such that

$$
J\left(S(T M)^{\perp}\right) \subset S(T M)
$$

where $S(T M)^{\perp}$ is the orthogonal complement of $S(T M)$ in the tangent bundle $T \bar{M}$ of $\bar{M}$, i. e., $T \bar{M}=S(T M) \oplus_{\text {orth }} S(T M)^{\perp}$. The notion of generic lightlike submanifold was introduced by Jin-Lee [8] at 2011 and later, studied by several authors (see [3-5]). The geometry of generic lightlike submanifold is an extension of that of lightlike hypersurface and half lightlike submanifold of codimension 2. Much of its theory will be immediately generalized in a formal way to general lightlike submanifolds.

A linear connection $\bar{\nabla}$ on a semi-Riemannian manifold $(\bar{M}, \bar{g})$ is called a semi-symmetric non-metric connection if it and its torsion $\bar{T}$ satisfy

$$
\left(\bar{\nabla}_{\bar{X}} \bar{g}\right)(\bar{Y}, \bar{Z})=-\theta(\bar{Y}) \bar{g}(\bar{X}, \bar{Z})-\theta(\bar{Z}) \bar{g}(\bar{X}, \bar{Y}),
$$

(C) Petrozavodsk State University, 2018 


$$
\bar{T}(\bar{X}, \bar{Y})=\theta(\bar{Y}) \bar{X}-\theta(\bar{X}) \bar{Y}
$$

where $\theta$ is a 1 -form on $\bar{M}$ associated with a smooth unit vector field $\zeta$ by $\theta(\bar{X})=\bar{g}(\bar{X}, \zeta)$. In the followings, we denote by $\bar{X}, \bar{Y}$ and $\bar{Z}$ the smooth vector fields on $\bar{M}$. The notion of semi-symmetric non-metric connection on a Riemannian manifold was introduced by Ageshe-Chafle [1].

Remark. Denote by $\widetilde{\nabla}$ a Levi-Civita connection of a semi-Riemannian manifold $(\bar{M}, \bar{g})$. It is known [7] that a linear connection $\bar{\nabla}$ on $\bar{M}$ is a semi-symmetric non-metric connection if and only if $\bar{\nabla}$ satisfies

$$
\bar{\nabla}_{\bar{X}} \bar{Y}=\widetilde{\nabla}_{\bar{X}} \bar{Y}+\theta(\bar{Y}) \bar{X}
$$

The object of present study is generic lightlike submanifolds of an indefinite Kaehler manifold with a semi-symmetric non-metric connection. First, we study the geometry of two types of generic lightlike submanifolds, named by recurrent and Lie recurrent, of such an indefinite Kaehler manifold. Next, we characterize generic lightlike submanifolds of an indefinite complex space form with a semi-symmetric non-metric connection.

2. Semi-symmetric non-metric connections. Let $\bar{M}=(\bar{M}, \bar{g}, J)$ be an indedinite Kaeler manifold, where $\bar{g}$ is a semi-Riemannian metric and $J$ is an indefinite almost complex structure:

$$
J^{2} \bar{X}=-\bar{X}, \quad \bar{g}(J \bar{X}, J \bar{Y})=\bar{g}(\bar{X}, \bar{Y}), \quad\left(\widetilde{\nabla}_{\bar{X}} J\right) \bar{Y}=0 .
$$

Replacing the Levi-Civita connection $\widetilde{\nabla}$ by the semi-symmetric non-metric connection $\bar{\nabla}$ given by (4), the third equation of (5) is reduced to

$$
\left(\bar{\nabla}_{\bar{X}} J\right) \bar{Y}=\theta(J \bar{Y}) \bar{X}-\theta(\bar{Y}) J \bar{X} .
$$

Let $(M, g)$ be an $m$-dimensional lightlike submanifold of an indefinite Kaehler manifold $(\bar{M}, \bar{g})$ of dimension $(m+n)$. Then the radical distribution $\operatorname{Rad}(T M)$, defined by $\operatorname{Rad}(T M)=T M \cap T M^{\perp}$, of $M$ is a subbundle of the tangent bundle $T M$ and the normal bundle $T M^{\perp}$, of rank $r(1 \leqslant r \leqslant \min \{m, n\})$. In general, there exist two complementary non-degenerate distributions $S(T M)$ and $S\left(T M^{\perp}\right)$ of $\operatorname{Rad}(T M)$ in $T M$ and $T M^{\perp}$ respectively, which are called the screen distribution and the co-screen distribution of $M[2]$, such that

$$
T M=\operatorname{Rad}(T M) \oplus_{\text {orth }} S(T M), \quad T M^{\perp}=\operatorname{Rad}(T M) \oplus_{\text {orth }} S\left(T M^{\perp}\right),
$$


where $\oplus_{\text {orth }}$ denotes the orthogonal direct sum. Denote by $F(M)$ the algebra of smooth functions on $M$ and by $\Gamma(E)$ the $F(M)$ module of smooth sections of a vector bundle $E$ over $M$. Also denote by $(5)_{i}$ the $i$-th equation of (5). We use the same notations for any others. Let $X, Y, Z$ and $W$ be the vector fields on $M$, unless otherwise specified. We use the following range of indices:

$$
i, j, k, \ldots \in\{1, \ldots, r\}, \quad a, b, c, \ldots \in\{r+1, \ldots, n\} .
$$

Let $\operatorname{tr}(T M)$ and $\operatorname{tr}(T M)$ be complementary vector bundles to $T M$ in $T \bar{M}_{\mid M}$ and $T M^{\perp}$ in $S(T M)^{\perp}$ respectively and let $\left\{N_{1}, \ldots, N_{r}\right\}$ be a lightlike basis of $l \operatorname{tr}(T M)_{\mid \mathcal{U}}$, where $\mathcal{U}$ is a neighborhood of $M$, such that

$$
\bar{g}\left(N_{i}, \xi_{j}\right)=\delta_{i j}, \quad \bar{g}\left(N_{i}, N_{j}\right)=0,
$$

where $\left\{\xi_{1}, \ldots, \xi_{r}\right\}$ is a lightlike basis of $\operatorname{Rad}(T M)_{\mid \mathcal{u}}$. Then we have

$$
\begin{aligned}
T \bar{M} & =T M \oplus \operatorname{tr}(T M)=\{\operatorname{Rad}(T M) \oplus \operatorname{tr}(T M)\} \oplus_{\text {orth }} S(T M)= \\
& =\{\operatorname{Rad}(T M) \oplus \operatorname{lt}(T M)\} \oplus_{\text {orth }} S(T M) \oplus_{\text {orth }} S\left(T M^{\perp}\right) .
\end{aligned}
$$

A lightlike submanifold $M=\left(M, g, S(T M), S\left(T M^{\perp}\right)\right)$ of $\bar{M}$ is called

(1) $r$-lightlike submanifold if $1 \leqslant r<\min \{m, n\}$;

(2) co-isotropic submanifold if $1 \leqslant r=n<m$;

(3) isotropic submanifold if $1 \leqslant r=m<n$;

(4) totally lightlike submanifold if $1 \leqslant r=m=n$.

The above three classes (2) - (4) are particular cases of (1) as follows:

$$
S\left(T M^{\perp}\right)=\{0\}, \quad S(T M)=\{0\}, \quad S(T M)=S\left(T M^{\perp}\right)=\{0\}
$$

respectively. The geometry of $r$-lightlike submanifolds is more general than that of the other three types. Thus we consider only $r$-lightlike submanifolds $M$, with following quasi-orthonormal field of frames of $\bar{M}$ :

$$
\left\{\xi_{1}, \ldots, \xi_{r}, N_{1}, \ldots, N_{r}, F_{r+1}, \ldots, F_{m}, E_{r+1}, \ldots, E_{n}\right\}
$$

where $\left\{F_{A}\right\}$ and $\left\{E_{a}\right\}$ are orthonormal bases of $S(T M)$ and $S\left(T M^{\perp}\right)$, respectively. Denote $\epsilon_{a}=\bar{g}\left(E_{a}, E_{a}\right)$. Then $\epsilon_{a} \delta_{a b}=\bar{g}\left(E_{a}, E_{b}\right)$. 
Let $P$ be the projection morphism of $T M$ on $S(T M)$. Then the local Gauss-Weingarten formulae of $M$ and $S(T M)$ are given respectively by

$$
\begin{aligned}
& \bar{\nabla}_{X} Y=\nabla_{X} Y+\sum_{i=1}^{r} h_{i}^{\ell}(X, Y) N_{i}+\sum_{a=r+1}^{n} h_{a}^{s}(X, Y) E_{a} \\
& \bar{\nabla}_{X} N_{i}=-A_{N_{i}} X+\sum_{j=1}^{r} \tau_{i j}(X) N_{j}+\sum_{a=r+1}^{n} \rho_{i a}(X) E_{a} \\
& \bar{\nabla}_{X} E_{a}=-A_{E_{a}} X+\sum_{i=1}^{r} \lambda_{a i}(X) N_{i}+\sum_{b=r+1}^{n} \mu_{a b}(X) E_{b} \\
& \nabla_{X} P Y=\nabla_{X}^{*} P Y+\sum_{i=1}^{r} h_{i}^{*}(X, P Y) \xi_{i} \\
& \nabla_{X} \xi_{i}=-A_{\xi_{i}}^{*} X-\sum_{j=1}^{r} \sigma_{j i}(X) \xi_{j}
\end{aligned}
$$

where $\nabla$ and $\nabla^{*}$ are induced linear connections on $M$ and $S(T M)$ respectively, $h_{i}^{\ell}$ and $h_{a}^{s}$ are called the local second fundamental forms on $M, h_{i}^{*}$ are called the local second fundamental forms on $S(T M) . A_{N_{i}}, A_{E_{a}}$ and $A_{\xi_{i}}^{*}$ are called the shape operators, and $\tau_{i j}, \rho_{i a}, \lambda_{a i}, \mu_{a b}$ and $\sigma_{j i}$ are 1-forms on $M$. Using (2), (3) and (7), we see that

$$
\begin{aligned}
\left(\nabla_{X} g\right)(Y, Z) & =\sum_{i=1}^{r}\left\{h_{i}^{\ell}(X, Y) \eta_{i}(Z)+h_{i}^{\ell}(X, Z) \eta_{i}(Y)\right\}- \\
& -\theta(Y) g(X, Z)-\theta(Z) g(X, Y) \\
& T(X, Y)=\theta(Y) X-\theta(X) Y
\end{aligned}
$$

and both $h_{i}^{\ell}$ and $h_{a}^{s}$ are symmetric, where $\eta_{i}$ 's are 1-forms such that

$$
\eta_{i}(X)=\bar{g}\left(X, N_{i}\right) .
$$

In the sequel, denote by $\alpha_{i}, \beta_{i}$ and $\gamma_{a}$ the functions given by

$$
\alpha_{i}=\theta\left(\xi_{i}\right), \quad \beta_{i}=\theta\left(N_{i}\right), \quad \gamma_{a}=\theta\left(E_{a}\right) .
$$

As $h_{i}^{\ell}(X, Y)=\bar{g}\left(\bar{\nabla}_{X} Y, \xi_{i}\right)$ and $\epsilon_{a} h_{a}^{s}(X, Y)=\bar{g}\left(\bar{\nabla}_{X} Y, E_{a}\right)$, we know that $h_{i}^{\ell}$ and $h_{a}^{s}$ are independent of the choice of $S(T M)$. The above three types local second fundamental forms are related to their shape operators by

$$
h_{i}^{\ell}(X, Y)=g\left(A_{\xi_{i}}^{*} X, Y\right)-\sum_{k=1}^{r} h_{k}^{\ell}\left(X, \xi_{i}\right) \eta_{k}(Y)+\alpha_{i} g(X, Y),
$$




$$
\begin{aligned}
& \epsilon_{a} h_{a}^{s}(X, Y)=g\left(A_{E_{a}} X, Y\right)-\sum_{k=1}^{r} \lambda_{a k}(X) \eta_{k}(Y)+\gamma_{a} g(X, Y), \\
& h_{i}^{*}(X, P Y)=g\left(A_{N_{i}} X, P Y\right)+\eta_{i}(X) \theta(P Y)+\beta_{i} g(X, P Y) .
\end{aligned}
$$

Applying the operator $\bar{\nabla}_{X}$ to $g\left(\xi_{i}, \xi_{j}\right)=0, \bar{g}\left(\xi_{i}, E_{a}\right)=0, \bar{g}\left(N_{i}, N_{j}\right)=0$, $\bar{g}\left(N_{i}, E_{a}\right)=0, \bar{g}\left(E_{a}, E_{b}\right)=\epsilon \delta_{a b}$ and $\bar{g}\left(N_{i}, \xi_{j}\right)=\delta_{i j}$ by turns, we have

$$
\left\{\begin{array}{l}
h_{i}^{\ell}\left(X, \xi_{j}\right)+h_{j}^{\ell}\left(X, \xi_{i}\right)=0, \quad h_{a}^{s}\left(X, \xi_{i}\right)=-\epsilon_{a} \lambda_{a i}(X), \\
\eta_{j}\left(A_{N_{i}} X\right)+\eta_{i}\left(A_{N_{j}} X\right)=-\beta_{i} \eta_{j}(X)-\beta_{j} \eta_{i}(X), \\
\bar{g}\left(A_{E_{a}} X, N_{i}\right)=\epsilon_{a} \rho_{i a}(X)-\gamma_{a} \eta_{i}(X), \\
\epsilon_{b} \mu_{a b}+\epsilon_{a} \mu_{b a}=0, \quad \tau_{i j}(X)=\sigma_{i j}(X)+\alpha_{j} \eta_{i}(X) .
\end{array}\right.
$$

Furthermore, using $(17)_{1}$, we see that

$$
h_{i}^{\ell}\left(X, \xi_{i}\right)=0, \quad h_{i}^{\ell}\left(\xi_{j}, \xi_{k}\right)=0, \quad A_{\xi_{i}}^{*} \xi_{i}=0 .
$$

Definition 1. We say that a lightlike submanifold of a semi-Riemannian manifold is irrotational [9] if $\bar{\nabla}_{X} \xi_{i} \in \Gamma(T M)$ for all $i \in\{1, \cdots, r\}$.

Remark. From (7) and (17) $)_{2}$, the above definition is equivalent to

$$
h_{j}^{\ell}\left(X, \xi_{i}\right)=0, \quad h_{a}^{s}\left(X, \xi_{i}\right)=\lambda_{a i}(X)=0 .
$$

3. Structure equations. Let $M$ be a generic lightlike submanifold of $\bar{M}$. From (1) we show that $J(\operatorname{Rad}(T M)), J(\operatorname{lt}(T M))$ and $J\left(S\left(T M^{\perp}\right)\right)$ are subbundles of $S(T M)$. Thus there exist two non-degenerate almost complex distributions $H_{o}$ and $H$ with respect to $J$, i. e., $J\left(H_{o}\right)=H_{o}$ and $J(H)=H$, such that

$$
\begin{aligned}
S(T M) & =\{J(\operatorname{Rad}(T M)) \oplus J(\operatorname{ltr}(T M))\} \oplus_{\text {orth }} J\left(S\left(T M^{\perp}\right)\right) \oplus_{\text {orth }} H_{o}, \\
H & =\operatorname{Rad}(T M) \oplus_{\text {orth }} J(\operatorname{Rad}(T M)) \oplus_{\text {orth }} H_{o} .
\end{aligned}
$$

In this case, the tangent bundle $T M$ of $M$ is decomposed as follow:

$$
T M=H \oplus J(\operatorname{ltr}(T M)) \oplus_{\text {orth }} J\left(S\left(T M^{\perp}\right)\right) .
$$

Consider $r$-th local null vector fields $U_{i}$ and $V_{i},(n-r)$-th local non-null unit vector fields $W_{a}$, and their 1 -forms $u_{i}, v_{i}$ and $w_{a}$ defined by

$$
U_{i}=-J N_{i}, \quad V_{i}=-J \xi_{i}, \quad W_{a}=-J E_{a},
$$




$$
u_{i}(X)=g\left(X, V_{i}\right), \quad v_{i}(X)=g\left(X, U_{i}\right), \quad w_{a}(X)=\epsilon_{a} g\left(X, W_{a}\right) .
$$

Denote by $S$ the projection morphism of $T M$ on $H$ and by $F$ the tensor field of type $(1,1)$ globally defined on $M$ by $F=J \circ S$. Then $X$ is expressed as $X=S X+\sum_{i=1}^{r} u_{i}(X) U_{i}+\sum_{a=r+1}^{n} w_{a}(X) W_{a}$. Therefore,

$$
J X=F X+\sum_{i=1}^{r} u_{i}(X) N_{i}+\sum_{a=r+1}^{n} w_{a}(X) E_{a} .
$$

Applying $J$ to (22) and using $(5)_{1},(20)$ and (22), we have

$$
F^{2} X=-X+\sum_{i=1}^{r} u_{i}(X) U_{i}+\sum_{a=r+1}^{n} w_{a}(X) W_{a} .
$$

In the sequel, we say that $F$ is the structure tensor field of $M$.

Applying the operator $\bar{\nabla}_{X}$ to (20) $)_{1,2,3}$ and (22) by turns and using (6), (7) - (11), (14) - (16) and (20) - (22), we have

$$
\begin{aligned}
h_{j}^{\ell}\left(X, U_{i}\right)= & u_{j}\left(A_{N_{i}} X\right)+\beta_{i} u_{j}(X)=h_{i}^{*}\left(X, V_{j}\right)-\theta\left(V_{j}\right) \eta_{i}(X), \\
h_{a}^{s}\left(X, U_{i}\right)=w_{a}\left(A_{N_{i}} X\right)+\beta_{i} w_{a}(X)=\epsilon_{a}\left\{h_{i}^{*}\left(X, W_{a}\right)-\theta\left(W_{a}\right) \eta_{i}(X)\right\}, & h_{a}^{s}\left(X, V_{i}\right)=\epsilon_{a} h_{i}^{\ell}\left(X, W_{a}\right), \\
h_{j}^{\ell}\left(X, V_{i}\right)= & \left.h_{i}^{\ell}\left(X, V_{j}\right), \quad X, W_{a}\right)=\epsilon_{a} h_{a}^{s}\left(X, W_{b}\right), \\
\nabla_{X} U_{i}= & F\left(A_{N_{i}} X\right)+\sum_{j=1}^{r} \tau_{i j}(X) U_{j}+\sum_{a=r+1}^{n} \rho_{i a}(X) W_{a}+ \\
& +\beta_{i} F X+\theta\left(U_{i}\right) X, \\
\nabla_{X} V_{i}= & F\left(A_{\xi_{i}}^{*} X\right)-\sum_{j=1}^{r} \sigma_{j i}(X) V_{j}+\sum_{j=1}^{r} h_{j}^{\ell}\left(X, \xi_{i}\right) U_{j}- \\
& -\sum_{a=r+1}^{n} \epsilon_{a} \lambda_{a i}(X) W_{a}+\alpha_{i} F X+\theta\left(V_{i}\right) X, \\
\nabla_{X} W_{a}= & F\left(A_{E_{a}} X\right)+\sum_{i=1}^{r} \lambda_{a i}(X) U_{i}+\sum_{b=r+1}^{n} \mu_{a b}(X) W_{b}+ \\
+ & \gamma_{a} F X+\theta\left(W_{a}\right) X,
\end{aligned}
$$




$$
\begin{aligned}
\left(\nabla_{X} F\right)(Y) & =\sum_{i=1}^{r} u_{i}(Y) A_{N_{i}} X+\sum_{a=r+1}^{n} w_{a}(Y) A_{E_{a}} X- \\
& -\sum_{i=1}^{r} h_{i}^{\ell}(X, Y) U_{i}-\sum_{a=r+1}^{n} h_{a}^{s}(X, Y) W_{a}+ \\
& +\theta(J Y) X-\theta(Y) F X .
\end{aligned}
$$

\section{Recurrent and Lie recurrent structure tensors.}

Definition 2. The structure tensor field $F$ of $M$ is said to be recurrent [6] if there exists a 1 -form $\varpi$ on $M$ such that

$$
\left(\nabla_{X} F\right) Y=\varpi(X) F Y
$$

A generic lightlike submanifold $M$ of an indefinite Kaehler manifold $\bar{M}$ is called recurrent if it admits a recurrent structure tensor field $F$.

Theorem 1. Let $M$ be a recurrent lightlike submanifold of an indefinite Kaehler manifold $\bar{M}$ with a semi-symmetric non-metric connection. Then

(1) $F$ is parallel with respect to the induced connection $\nabla$ on $M$,

(2) $M$ is irrotational and the 1-forms $\rho_{i a}$ satisfy $\rho_{i a}=0$,

(3) the 1-form $\theta$ vanishes on $T M$,

(4) $H, J(\operatorname{lt}(T M))$ and $J\left(S\left(T M^{\perp}\right)\right)$ are parallel distributions on $M$,

(5) $M$ is locally a product manifold $M_{r} \times M_{n-r} \times M^{\sharp}$, where $M_{r}, M_{n-r}$ and $M^{\sharp}$ are leaves of $J(\operatorname{ltr}(T M)), J\left(S\left(T M^{\perp}\right)\right)$ and $H$, respectively.

\section{Proof.}

(1) From the above definition and (27), we obtain

$$
\begin{aligned}
& \varpi(X) F Y=\sum_{i=1}^{r} u_{i}(Y) A_{N_{i}} X+\sum_{a=r+1}^{n} w_{a}(Y) A_{E_{a}} X- \\
& -\sum_{i=1}^{r} h_{i}^{\ell}(X, Y) U_{i}-\sum_{a=r+1}^{n} h_{a}^{s}(X, Y) W_{a}+\theta(J Y) X-\theta(Y) F X .
\end{aligned}
$$

Replacing $Y$ by $\xi_{j}$ to this and using the fact that $F \xi_{j}=-V_{j}$, we get

$$
\varpi(X) V_{j}=\sum_{k=1}^{r} h_{k}^{\ell}\left(X, \xi_{j}\right) U_{k}+\sum_{b=r+1}^{n} h_{b}^{s}\left(X, \xi_{j}\right) W_{b}+\theta\left(V_{j}\right) X+\alpha_{j} F X .
$$


Taking the scalar product with $N_{i}$ to (30), we obtain

$$
\theta\left(V_{j}\right) \eta_{i}(X)+\alpha_{j} v_{i}(X)=0 .
$$

Taking $X=V_{i}$ and $X=\xi_{i}$ to this equation by turns, we have

$$
\alpha_{i}=0, \quad \theta\left(V_{i}\right)=0,
$$

for all $i$. Taking the scalar product with $U_{j}$ to (30), we get $\varpi=0$. Thus $F$ is parallel with respect to the induced connection $\nabla$ on $M$.

(2) Taking the scalar product with $V_{i}$ and $W_{a}$ to (30) such that $\varpi=\alpha_{j}=\theta\left(V_{j}\right)=0$, we obtain $h_{i}^{\ell}\left(X, \xi_{j}\right)=0$ and $h_{a}^{s}\left(X, \xi_{j}\right)=0$. Thus $M$ is irrotational by Remark in Section 2.

Replacing $Y$ by $W_{a}$ to (29) such that $\varpi=0$, we have

$$
A_{E_{a}} X=\sum_{i=1}^{r} h_{i}^{\ell}\left(X, W_{a}\right) U_{i}+\sum_{b=r+1}^{n} h_{b}^{s}\left(X, W_{a}\right) W_{b}-\gamma_{a} X+\theta\left(W_{a}\right) F X .
$$

Taking the scalar product with $N_{i}$ and $U_{i}$ to this equation by turns and using (15), (17) 4 , we obtain

$$
\epsilon_{a} \rho_{i a}(X)=\theta\left(W_{a}\right) v_{i}(X), \quad \epsilon_{a} h_{a}^{s}\left(X, U_{i}\right)=-\theta\left(W_{a}\right) \eta_{i}(X) .
$$

Replacing $X$ by $\xi_{i}$ to $(33)_{2}$ and using the fact that $h_{a}^{s}\left(\xi_{i}, U_{i}\right)=0$, we get $\theta\left(W_{a}\right)=0$. From this result and $(33)_{1}$, we see that $\rho_{i a}=0$. Thus

$$
\theta\left(W_{a}\right)=0, \quad \rho_{i a}=0, \quad h_{a}^{s}\left(X, U_{i}\right)=0 .
$$

(3) Replacing $Y$ by $U_{i}$ to (29) such that $\varpi=0$, we have

$$
A_{N_{i}} X=\sum_{k=1}^{r} h_{k}^{\ell}\left(X, U_{i}\right) U_{k}+\sum_{a=r+1}^{n} h_{a}^{s}\left(X, U_{i}\right) W_{a}-\beta_{i} X+\theta\left(U_{i}\right) F X .
$$

Taking the scalar product with $N_{j}$ and $U_{j}$ to this by turns, we get

$$
\begin{aligned}
\eta_{j}\left(A_{N_{i}} X\right) & =-\beta_{i} \eta_{j}(X)-\theta\left(U_{i}\right) v_{j}(X), \\
g\left(A_{N_{i}} X, U_{j}\right) & =-\beta_{i} v_{j}(X)-\theta\left(U_{i}\right) \eta_{j}(X) .
\end{aligned}
$$

Taking $i=j$ to $(36)_{1}$ and using $(17)_{3}$, we get $\theta\left(U_{i}\right) v_{i}(X)=0$. It follows that $\theta\left(U_{i}\right)=0$. Using $(16),(36)_{2}$ reduces $h_{i}^{*}\left(X, U_{j}\right)=0$. Thus

$$
\theta\left(U_{i}\right)=0, \quad h_{i}^{*}\left(X, U_{j}\right)=0 .
$$


Replacing $X$ by $\xi_{j}$ to (29) and using $M$ is irrotational, we get

$$
\sum_{i=1}^{r} u_{i}(Y) A_{N_{i}} \xi_{j}+\sum_{a=r+1}^{n} w_{a}(Y) A_{E_{a}} \xi_{j}+\theta(J Y) \xi_{j}+\theta(Y) V_{j}=0 .
$$

Taking the scalar product with $U_{j}$ to this equation, we have

$$
\sum_{i=1}^{r} u_{i}(Y) \bar{g}\left(A_{N_{i}} \xi_{j}, U_{j}\right)+\sum_{a=r+1}^{n} w_{a}(Y) \bar{g}\left(A_{E_{a}} \xi_{j}, U_{j}\right)+\theta(Y)=0
$$

Taking $Y=U_{i}$ and $Y=W_{a}$ by turns and using (34) 1 and $(37)_{1}$, we get

$$
\bar{g}\left(A_{N_{i}} \xi_{j}, U_{j}\right)=0, \quad \bar{g}\left(A_{E_{a}} \xi_{j}, U_{j}\right)=0 .
$$

Consequently, (38) is reduced to $\theta(X)=0$. Thus $\theta$ vanishes on $T M$.

(4) Using (2), (11), (14), (15), (22), (26) and (27), we get

$$
\left\{\begin{array}{l}
g\left(\nabla_{X} \xi_{i}, V_{j}\right)=-h_{i}^{\ell}\left(X, V_{j}\right)+\alpha_{i} u_{j}(X), \\
g\left(\nabla_{X} \xi_{i}, W_{a}\right)=-h_{i}^{\ell}\left(X, W_{a}\right)+\epsilon_{a} \alpha_{i} w_{a} w(X), \\
g\left(\nabla_{X} V_{i}, V_{j}\right)=h_{j}^{\ell}\left(X, \xi_{i}\right)+\theta\left(V_{i}\right) u_{j}(X), \\
g\left(\nabla_{X} V_{i}, W_{a}\right)=-\lambda_{a i}(X)+\epsilon_{a} \theta\left(V_{i}\right) w_{a}(X), \\
g\left(\nabla_{X} Z, V_{j}\right)=h_{j}^{\ell}(X, F Z)+\theta(Z) u_{j}(X), \\
g\left(\nabla_{X} Z, W_{a}\right)=\epsilon_{a}\left\{h_{a}^{s}(X, F Z)+\theta(Z) w_{a}(X)\right\},
\end{array}\right.
$$

for any $X \in \Gamma(T M)$ and $Z \in \Gamma\left(H_{o}\right)$. Taking $Y=V_{j}$ and $Y=F Z$, $Z \in \Gamma\left(H_{o}\right)$ to (29) by turns and using (31) and the facts that $\theta=0$ on $T M, u_{i}(F Z)=w_{a}(F Z)=0$ and $J F Z=F^{2} Z=-Z$, we have

$$
\begin{array}{cc}
h_{i}^{\ell}\left(X, V_{j}\right)=0, & h_{a}^{s}\left(X, V_{j}\right)=h_{j}^{\ell}\left(X, W_{a}\right)=0, \\
h_{i}^{\ell}(X, F Z)=0, & h_{a}^{s}(X, F Z)=0 .
\end{array}
$$

Using (31), (40), (41) and $\lambda_{a i}=0,(39)$ are equivalent to

$$
\nabla_{X} Y \in \Gamma(H), \quad \forall X \in \Gamma(T M), \quad \forall Y \in \Gamma(H) .
$$

It follows that $H$ is a parallel distribution on $M$.

Applying $F$ to (32) and (35) and using (34) 1 and (37) $)_{1}$, we get

$$
F\left(A_{N_{i}} X\right)=-\beta_{i} F X, \quad F\left(A_{E_{a}} X\right)=-\gamma_{a} F X .
$$


Using these results together with (34), (37) and $\lambda_{a i}=0,(25)$ and (27) reduce to

$$
\begin{array}{ll}
\nabla_{X} U_{i}=\sum_{j=1}^{r} \tau_{i j}(X) U_{j}, & \nabla_{X} U_{i} \in \Gamma(J(\operatorname{ltr}(T M))), \\
\nabla_{X} W_{a}=\sum_{b=r+1}^{n} \mu_{a b} W_{b}, & \nabla_{X} W_{a} \in \Gamma\left(J\left(S\left(T M^{\perp}\right)\right)\right) .
\end{array}
$$

Thus $J(\operatorname{lt}(T M))$ and $J\left(S\left(T M^{\perp}\right)\right)$ are also parallel distributions on $M$.

(5) As $H, J(\operatorname{lt}(T M))$ and $J\left(S\left(T M^{\perp}\right)\right)$ are parallel distributions and satisfy (19), by the decomposition theorem of de Rham [10], $M$ is locally a product manifold $M_{r} \times M_{n-r} \times M^{\sharp}$, where $M_{r}, M_{n-r}$ and $M^{\sharp}$ are leaves of the distributions $J(\operatorname{lt}(T M)), J\left(S\left(T M^{\perp}\right)\right)$ and $H$ respectively.

Definition 3. The structure tensor field $F$ of $M$ is said to be Lie recurrent [6] if there exists a 1-form $\vartheta$ on $M$ such that

$$
\left(\mathcal{L}_{X} F\right) Y=\vartheta(X) F Y,
$$

where $\mathcal{L}_{X}$ denotes the Lie derivative on $M$ with respect to $X$, that is,

$$
\left(\mathcal{L}_{X} F\right) Y=[X, F Y]-F[X, Y] .
$$

In the case $\vartheta=0$, i. e., $\mathcal{L}_{X} F=0$, we say that $F$ is Lie parallel. A generic lightlike submanifold $M$ of an indefinite Kaehler manifold $\bar{M}$ is called Lie recurrent if it admits a Lie recurrent structure tensor field $F$.

Theorem 2. Let $M$ be a Lie recurrent generic lightlike submanifold of an indefinite Kaehler manifold $\bar{M}$ with a semi-symmetric non-metric connection. Then

(1) $F$ is Lie parallel,

(2) $\tau_{i j}$ and $\rho_{i a}$ satisfy $\tau_{i j}(F X)=0$ and $\rho_{i a}(F X)=0$. Moreover,

$$
\tau_{i j}(X)=\sum_{k=1}^{r} u_{k}(X) g\left(A_{N_{k}} V_{j}, N_{i}\right) .
$$

\section{Proof.}

(1) Using (13), (22), (28) and (44), we obtain 


$$
\begin{aligned}
\vartheta(X) F Y=- & \nabla_{F Y} X+F \nabla_{Y} X+ \\
& +\sum_{i=1}^{r} u_{i}(Y) A_{N_{i}} X+\sum_{a=r+1}^{n} w_{a}(Y) A_{E_{a}} X- \\
& -\sum_{i=1}^{r} h_{i}^{\ell}(X, Y) U_{i}-\sum_{a=r+1}^{n} h_{a}^{s}(X, Y) W_{a}+ \\
& +\left\{\sum_{i=1}^{r} \beta_{i} u_{i}(Y)+\sum_{a=r+1}^{n} \gamma_{a} w_{a}(Y)\right\} X .
\end{aligned}
$$

Replacing $Y$ by $\xi_{j}$ and $Y$ by $V_{j}$ to (45) respectively, we have

$$
\begin{gathered}
-\vartheta(X) V_{j}=\nabla_{V_{j}} X+F \nabla_{\xi_{j}} X- \\
\quad-\sum_{i=1}^{r} h_{i}^{\ell}\left(X, \xi_{j}\right) U_{i}-\sum_{a=r+1}^{n} h_{a}^{s}\left(X, \xi_{j}\right) W_{a}, \\
\vartheta(X) \xi_{j}=-\nabla_{\xi_{j}} X+F \nabla_{V_{j}} X- \\
-\sum_{i=1}^{r} h_{i}^{\ell}\left(X, V_{j}\right) U_{i}-\sum_{a=r+1}^{n} h_{a}^{s}\left(X, V_{j}\right) W_{a} .
\end{gathered}
$$

Taking the scalar product with $U_{i}$ to $(46)$ and $N_{i}$ to (47), we get

$$
\begin{aligned}
-\delta_{i j} \vartheta(X) & =g\left(\nabla_{V_{j}} X, U_{i}\right)-\bar{g}\left(\nabla_{\xi_{j}} X, N_{i}\right), \\
\delta_{i j} \vartheta(X) & =g\left(\nabla_{V_{j}} X, U_{i}\right)-\bar{g}\left(\nabla_{\xi_{j}} X, N_{i}\right),
\end{aligned}
$$

respectively. It follows that $\vartheta=0$. Thus $F$ is Lie parallel.

(2) Taking the scalar product with $N_{i}$ to (46) such that $X=W_{a}$ and using (15), (17) 4 and (27), we get $h_{a}^{s}\left(U_{i}, V_{j}\right)=\rho_{i a}\left(\xi_{j}\right)$. Also, taking the scalar product with $W_{a}$ to (47) such that $X=U_{i}$ and using (25), we have $h_{a}^{s}\left(U_{i}, V_{j}\right)=-\rho_{i a}\left(\xi_{j}\right)$. Thus $\rho_{i a}\left(\xi_{j}\right)=0$ and $h_{a}^{s}\left(U_{i}, V_{j}\right)=0$.

Taking the scalar product with $U_{i}$ to (46) with $X=W_{a}$ and using (15), (17) $)_{2,4}$ and (27), we get $\epsilon_{a} \rho_{i a}\left(V_{j}\right)=\lambda_{a j}\left(U_{i}\right)$. Also, taking the scalar product with $W_{a}$ to (46) such that $X=U_{i}$ and using (17) $)_{2}$ and (25), we get $\epsilon_{a} \rho_{i a}\left(V_{j}\right)=-\lambda_{a j}\left(U_{i}\right)$. Thus $\rho_{i a}\left(V_{j}\right)=0$ and $\lambda_{a j}\left(U_{i}\right)=0$.

Taking the scalar product with $V_{i}$ to (46) such that $X=W_{a}$ and using $(17)_{2},(24)_{4}$ and $(27)$, we obtain $\lambda_{a i}\left(V_{j}\right)=-\lambda_{a j}\left(V_{i}\right)$. Also, taking the scalar product with $W_{a}$ to (46) such that $X=V_{i}$ and using (17) $)_{2}$ and (26), we have $\lambda_{a i}\left(V_{j}\right)=\lambda_{a j}\left(V_{i}\right)$. Thus we obtain $\lambda_{a i}\left(V_{j}\right)=0$. 
Taking the scalar product with $W_{a}$ to (46) such that $X=\xi_{i}$ and using (11), (14) and $(17)_{2}$, we get $h_{i}^{\ell}\left(V_{j}, W_{a}\right)=\lambda_{a i}\left(\xi_{j}\right)$. Also, taking the scalar product with $V_{i}$ to (47) such that $X=W_{a}$ and using (27), we have $h_{i}^{\ell}\left(V_{j}, W_{a}\right)=-\lambda_{a i}\left(\xi_{j}\right)$. Thus $\lambda_{a i}\left(\xi_{j}\right)=0$ and $h_{i}^{\ell}\left(V_{j}, W_{a}\right)=0$.

Summarizing the above results, we obtain

$$
\begin{gathered}
\rho_{i a}\left(\xi_{j}\right)=0, \quad \rho_{i a}\left(V_{j}\right)=0, \quad \lambda_{a i}\left(U_{j}\right)=0, \quad \lambda_{a i}\left(V_{j}\right)=0, \quad \lambda_{a i}\left(\xi_{j}\right)=0, \\
h_{a}^{s}\left(U_{i}, V_{j}\right)=h_{j}^{\ell}\left(U_{i}, W_{a}\right)=0, \quad h_{i}^{\ell}\left(V_{j}, W_{a}\right)=h_{a}^{s}\left(V_{j}, V_{i}\right)=0 .
\end{gathered}
$$

Taking the scalar product with $N_{i}$ to (45) and using (17) $)_{4}$, we have

$$
\begin{aligned}
-\bar{g}\left(\nabla_{F Y} X, N_{i}\right)+g\left(\nabla_{Y} X, U_{i}\right)+\sum_{a=r+1}^{n} \epsilon_{a} w_{a}(Y) \rho_{i a}(X)+ \\
+\sum_{k=1}^{r} u_{k}(Y)\left\{\bar{g}\left(A_{N_{k}} X, N_{i}\right)+\beta_{k} \eta_{i}(X)\right\}=0 .
\end{aligned}
$$

Taking $X=\xi_{j}$ and $Y=U_{k}$ to (50) and using (11) and (14), we have

$$
h_{j}^{\ell}\left(U_{k}, U_{i}\right)=g\left(A_{N_{k}} \xi_{j}, N_{i}\right)+\beta_{k} \delta_{i j} .
$$

As $h_{j}^{\ell}$ is symmetric, applying $(24)_{1}\left\{\right.$ take $\left.X=U_{i}\right\}$ to (51), we obtain

$$
h_{k}^{*}\left(U_{i}, V_{j}\right)=h_{j}^{\ell}\left(U_{i}, U_{k}\right)=g\left(A_{N_{k}} \xi_{j}, N_{i}\right)+\beta_{k} \delta_{i j} .
$$

On the other hand, applying $(24)_{1}\left\{\right.$ take $\left.X=U_{k}\right\}$ to (51), we obtain

$$
h_{i}^{*}\left(U_{k}, V_{j}\right)=g\left(A_{N_{k}} \xi_{j}, N_{i}\right)+\beta_{k} \delta_{i j} .
$$

Exchanging $i$ by $k$ and $k$ by $i$ to this equation and using $(17)_{3}$, we have

$$
h_{k}^{*}\left(U_{i}, V_{j}\right)=\bar{g}\left(A_{N_{i}} \xi_{j}, N_{k}\right)+\beta_{i} \delta_{k j}=-\bar{g}\left(A_{N_{k}} \xi_{j}, N_{i}\right)-\beta_{k} \delta_{i j} .
$$

Comparing (52) with (53), we obtain

$$
g\left(A_{N_{k}} \xi_{j}, N_{i}\right)+\beta_{k} \delta_{i j}=0 .
$$

Replacing $X$ by $\xi_{j}$ to (50) and using (11), (14), (17) $)_{6},(49)_{1}$ and (54), we get

$$
h_{j}^{\ell}\left(X, U_{i}\right)=\tau_{i j}(F X) .
$$

Taking $X=V_{j}$ to (50) and using $(17)_{6},(26)$ and $(49)_{2}$, we have

$$
h_{j}^{\ell}\left(F X, U_{i}\right)+\tau_{i j}(X)=\sum_{k=1}^{r} u_{k}(X) \bar{g}\left(A_{N_{k}} V_{j}, N_{i}\right) .
$$


Taking $X=U_{i}$ to (45) and using (16), (23), (24) 1,2 $_{2}$ and (25), we get

$$
\begin{aligned}
\sum_{k=1}^{r} u_{k}(Y) & A_{N_{k}} U_{i}+\sum_{a=r+1}^{n} w_{a}(Y) A_{E a} U_{i}-A_{N_{i}} Y- \\
& -F\left(A_{N_{i}} F Y\right)-\sum_{j=1}^{r} \tau_{i j}(F Y) U_{j}-\sum_{a=r+1}^{n} \rho_{i a}(F Y) W_{a}+ \\
+ & \left\{\sum_{j=1}^{r} \beta_{j} u_{j}(Y)+\sum_{a=r+1}^{n} \gamma_{a} w_{a}(Y)\right\} U_{i}-\beta_{i}\left\{F^{2} Y+Y\right\}=0 .
\end{aligned}
$$

Taking scalar product with $V_{j}$ to (57) and using (54), we get

$$
h_{j}^{\ell}\left(X, U_{i}\right)=-\tau_{i j}(F X) .
$$

Comparing this equation with (55), we obtain

$$
\tau_{i j}(F X)=0, \quad h_{j}^{\ell}\left(X, U_{i}\right)=0 .
$$

Using $(58)_{2}$, the equation (56) reduced to

$$
\tau_{i j}(X)=\sum_{k=1}^{r} u_{k}(X) \bar{g}\left(A_{N_{k}} V_{j}, N_{i}\right)
$$

Taking the scalar product with $U_{j}$ to $(57)$ and then, taking $Y=W_{a}$ and using (15), (16) and (24) 2 , we have

$$
h_{i}^{*}\left(W_{a}, U_{j}\right)=\epsilon_{a} h_{a}^{s}\left(U_{i}, U_{j}\right)=\epsilon_{a} h_{a}^{s}\left(U_{j}, U_{i}\right)=h_{i}^{*}\left(U_{j}, W_{a}\right) .
$$

Taking the scalar product with $W_{a}$ to (57) and using (23), we have $\epsilon_{a} \rho_{i a}(F Y)=-h_{i}^{*}\left(Y, W_{a}\right)+\sum_{k=1}^{r} u_{k}(Y) h_{k}^{*}\left(U_{i}, W_{a}\right)+\sum_{b=r+1}^{n} \epsilon_{b} w_{b}(Y) h_{b}^{s}\left(U_{i}, W_{a}\right)$ by (15) and (16). Taking the scalar product with $U_{i}$ to (45) such that $X=W_{a}$ and using $(17)_{4},(23),(24)_{2}$ and (60), we get

$$
\epsilon_{a} \rho_{i a}(F Y)=h_{i}^{*}\left(Y, W_{a}\right)-\sum_{k=1}^{r} u_{k}(Y) h_{k}^{*}\left(U_{i}, W_{a}\right)-\sum_{b=r+1}^{n} \epsilon_{b} w_{b}(Y) h_{b}^{s}\left(U_{i}, W_{a}\right) .
$$

Comparing the last two equations, we obtain $\rho_{i a}(F Y)=0$. 


\section{Indefinite complex space forms.}

Definition 4. An indefinite complex space form $\bar{M}(c)$ is an indefinite Kaehler manifold of constant holomorphic sectional curvature $c$ such that

$$
\begin{aligned}
\widetilde{R}(\bar{X}, \bar{Y}) \bar{Z}=\frac{c}{4}\{\bar{g}(\bar{Y}, \bar{Z}) \bar{X}-\bar{g}(\bar{X}, \bar{Z}) \bar{Y}+\bar{g}(J \bar{Y}, \bar{Z}) J \bar{X}- & \\
& -\bar{g}(J \bar{X}, \bar{Z}) J \bar{Y}+2 \bar{g}(\bar{X}, J \bar{Y}) J \bar{Z}\},
\end{aligned}
$$

where $\widetilde{R}$ is the curvature tensor of the Levi-Civita connection $\widetilde{\nabla}$ on $\bar{M}$.

Let $\bar{R}$ be the curvature tensor of the semi-symmetric non-metric connection $\bar{\nabla}$ on $\bar{M}$. By directed calculations from (3) and (4), we get

$$
\bar{R}(\bar{X}, \bar{Y}) \bar{Z}=\widetilde{R}(\bar{X}, \bar{Y}) \bar{Z}+\left(\bar{\nabla}_{\bar{X}} \theta\right)(\bar{Z}) \bar{Y}-\left(\bar{\nabla}_{\bar{Y}} \theta\right)(\bar{Z}) \bar{X} .
$$

Denote by $R$ and $R^{*}$ the curvature tensors of the induced linear connections $\nabla$ and $\nabla^{*}$ on $M$ and $S(T M)$ respectively. Using the GaussWeingarten formulae, we obtain Gauss equations for $M$ and $S(T M)$ respectively:

$$
\begin{gathered}
\bar{R}(X, Y) Z=R(X, Y) Z+\sum_{i=1}^{r}\left\{h_{i}^{\ell}(X, Z) A_{N_{i}} Y-h_{i}^{\ell}(Y, Z) A_{N_{i}} X\right\}+ \\
+\sum_{a=r+1}^{n}\left\{h_{a}^{s}(X, Z) A_{E_{a}} Y-h_{a}^{s}(Y, Z) A_{E_{a}} X\right\}+\sum_{i=1}^{r}\left\{\left(\nabla_{X} h_{i}^{\ell}\right)(Y, Z)-\right. \\
\quad-\left(\nabla_{Y} h_{i}^{\ell}\right)(X, Z)+\sum_{j=1}^{r}\left[\tau_{j i}(X) h_{j}^{\ell}(Y, Z)-\tau_{j i}(Y) h_{j}^{\ell}(X, Z)\right]+ \\
\quad+\sum_{a=r+1}^{n}\left[\lambda_{a i}(X) h_{a}^{s}(Y, Z)-\lambda_{a i}(Y) h_{a}^{s}(X, Z)\right]-\theta(X) h_{i}^{\ell}(Y, Z)+ \\
\left.+\theta(Y) h_{i}^{\ell}(X, Z)\right\} N_{i}+\sum_{a=r+1}^{n}\left\{\left(\nabla_{X} h_{a}^{s}\right)(Y, Z)-\left(\nabla_{Y} h_{a}^{s}\right)(X, Z)+\right. \\
+\sum_{i=1}^{r}\left[\rho_{i a}(X) h_{i}^{\ell}(Y, Z)-\rho_{i a}(Y) h_{i}^{\ell}(X, Z)\right]+\sum_{b=r+1}^{n}\left[\mu_{b a}(X) h_{b}^{s}(Y, Z)-\right. \\
\left.\left.\quad-\mu_{b a}(Y) h_{b}^{s}(X, Z)\right]-\theta(X) h_{a}^{s}(Y, Z)+\theta(Y) h_{a}^{s}(X, Z)\right\} E_{a},
\end{gathered}
$$

$$
\begin{aligned}
R(X, Y) P Z & =R^{*}(X, Y) P Z+ \\
& +\sum_{i=1}^{r}\left\{h_{i}^{*}(X, P Z) A_{\xi_{i}}^{*} Y-h_{i}^{*}(Y, P Z) A_{\xi_{i}} X\right\}+
\end{aligned}
$$




$$
\begin{aligned}
& +\sum_{i=1}^{r}\left\{\left(\nabla_{X} h_{i}^{*}\right)(Y, P Z)-\left(\nabla_{Y} h_{i}^{*}\right)(X, P Z)+\right. \\
& +\sum_{k=1}^{r}\left[\sigma_{i k}(Y) h_{k}^{*}(X, P Z)-\sigma_{i k}(X) h_{k}^{*}(Y, P Z)\right]- \\
& \left.\quad-\theta(X) h_{i}^{*}(Y, P Z)+\theta(Y) h_{i}^{*}(X, P Z)\right\} \xi_{i} .
\end{aligned}
$$

Comparing the tangential, lightlike transversal and radical components of the two equations (62) and (63) and using (22), we get

$$
\begin{gathered}
R(X, Y) Z=\sum_{i=1}^{r}\left\{h_{i}^{\ell}(Y, Z) A_{N_{i}} X-h_{i}^{\ell}(X, Z) A_{N_{i}} Y\right\}+ \\
\quad+\sum_{a=r+1}^{n}\left\{h_{a}^{s}(Y, Z) A_{E_{a}} X-h_{a}^{s}(X, Z) A_{E_{a}} Y\right\}+ \\
\quad+\left(\bar{\nabla}_{X} \theta\right)(Z) Y-\left(\bar{\nabla}_{Y} \theta\right)(Z) X+ \\
\quad+\frac{c}{4}\{g(Y, Z) X-g(X, Z) Y+\bar{g}(J Y, Z) F X- \\
\quad-\bar{g}(J X, Z) F Y+2 \bar{g}(X, J Y) F Z\}, \quad(65) \\
\left(\nabla_{X} h_{i}^{\ell}\right)(Y, Z)-\left(\nabla_{Y} h_{i}^{\ell}\right)(X, Z)+\sum_{k=1}^{r}\left\{h_{k}^{\ell}(Y, Z) \tau_{k i}(X)-\right. \\
\left.-h_{k}^{\ell}(X, Z) \tau_{k i}(Y)\right\}+\sum_{a=r+1}^{n}\left\{h_{a}^{s}(Y, Z) \lambda_{a i}(X)-h_{a}^{s}(X, Z) \lambda_{a i}(Y)\right\}- \\
-h_{i}^{\ell}(Y, Z) \theta(X)+\theta(Y) h_{i}^{\ell}(X, Z) \theta(Y)= \\
=\frac{c}{4}\left\{u_{i}(X) \bar{g}(J Y, Z)-u_{i}(Y) \bar{g}(J X, Z)+2 u_{i}(Z) \bar{g}(X, J Y)\right\} \\
-\sum_{a=r+1}^{n}\left\{h_{a}^{s}(Y, P Z) \eta_{i}\left(A_{E_{a}} X\right)-h_{a}^{s}(X, P Z) \eta_{i}\left(A_{E_{a}} Y\right)\right\}-h_{i}^{*}(Y, P Z) \theta(X)+ \\
\left(\nabla_{X} h_{i}^{*}\right)(Y, P Z)-\left(\nabla_{Y} h_{i}^{*}\right)(X, P Z)- \\
-\sum_{k=1}^{r}\left\{h_{k}^{*}(Y, P Z) \sigma_{i k}(X)-h_{k}^{*}(X, P Z) \sigma_{i k}(Y)\right\}- \\
\sum_{k=1}^{r}\left\{h_{k}^{\ell}(Y, P Z) \eta_{i}\left(A_{N_{k}} X\right)-h_{k}^{\ell}(X, P Z) \eta_{i}\left(A_{N_{k}} Y\right)\right\}-
\end{gathered}
$$




$$
\begin{array}{r}
+h_{i}^{*}(X, P Z) \theta(Y)-\left(\bar{\nabla}_{X} \theta\right)(P Z) \eta_{i}(Y)+\left(\bar{\nabla}_{Y} \theta\right)(P Z) \eta_{i}(X)= \\
=\frac{c}{4}\left\{g(Y, P Z) \eta_{i}(X)-g(X, P Z) \eta_{i}(Y)+v_{i}(X) \bar{g}(J Y, P Z)-\right. \\
\left.-v_{i}(Y) \bar{g}(J X, P Z)+2 v_{i}(P Z) \bar{g}(X, J Y)\right\}
\end{array}
$$

Theorem 3. Let $M$ be a generic lightlike submanifold of an indefinite complex space form $\bar{M}(c)$ with a semi-symmetric non-metric connection. If one of the following four statements

(1) $M$ is recurrent,

(2) $M$ is Lie recurrent,

(3) $U_{i}$ is parallel with respect to the connection $\nabla$, or

(4) $V_{i}$ is parallel with respect to the connection $\nabla$ is satisfied, then $\bar{M}(c)$ is flat, i. e., $c=0$.

Proof. (1) By Theorem 1, we get $\rho_{i a}=0$ and $\theta=0$ on $T M$, and we have $(34)_{3},(36)$ and $(37)_{1,2}$. From $(36)_{1}$ and $(37)_{1}: \theta\left(U_{i}\right)=0$, we obtain

$$
\eta_{i}\left(A_{N_{j}} X\right)=-\beta_{j} \eta_{i}(X)
$$

Applying $\bar{\nabla}_{X}$ to $\theta\left(U_{i}\right)=0$ and using $(7),(34)_{3}$ and $\theta_{\left.\right|_{T M}}=0$, we have

$$
\left(\bar{\nabla}_{X} \theta\right)\left(U_{i}\right)=-\sum_{k=1}^{r} \beta_{k} h_{k}^{\ell}\left(X, U_{i}\right) .
$$

Applying $\nabla_{X}$ to $(37)_{2}: h_{i}^{*}\left(Y, U_{j}\right)=0$ and using $(42)_{1}$, we obtain

$$
\left(\nabla_{X} h_{i}^{*}\right)\left(Y, U_{j}\right)=0 .
$$

Taking $P Z=U_{j}$ to $(67)$ and using $(34)_{3},(37)_{2}(68)$ and (69), we have

$$
\frac{c}{4}\left\{v_{j}(Y) \eta_{i}(X)-v_{j}(X) \eta_{i}(Y)+v_{i}(Y) \eta_{j}(X)-v_{i}(X) \eta_{j}(Y)\right\}=0 .
$$

Taking $X=\xi_{i}$ and $Y=V_{j}$, we have $c=0$ and $\bar{M}(c)$ is flat.

(2) Taking $X=\xi_{j}$ to (14) and using (18) 2 and $h_{i}^{\ell}$ is symmetric, we get $h_{i}^{\ell}\left(X, \xi_{j}\right)=g\left(A_{\xi_{i}}^{*} \xi_{j}, X\right)$. From this result and $(17)_{1}$, we obtain $g\left(A_{\xi_{i}}^{*} \xi_{j}+\right.$ $\left.+A_{\xi_{j}}^{*} \xi_{i}, X\right)=0$. As $S(T M)$ is non-degenerate, we get $A_{\xi_{i}}^{*} \xi_{j}=-A_{\xi_{j}}^{*} \xi_{i}$. Thus $A_{\xi_{i}}^{*} \xi_{j}$ is skew-symmetric with respect to $i$ and $j$.

In the case $M$ is Lie recurrent, taking $Y=U_{j}$ to (57), we have

$$
A_{N_{j}} U_{i}+\beta_{j} U_{i}=A_{N_{i}} U_{j}+\beta_{i} U_{j}
$$


Applying $F$ to this equation, we have $F\left(A_{N_{j}} U_{i}\right)=F\left(A_{N_{i}} U_{j}\right)$. Thus $F\left(A_{N_{i}} U_{j}\right)$ is symmetric with respect to $i$ and $j$. Therefore, we obtain

$$
h_{i}^{\ell}\left(\xi_{j}, F\left(A_{N_{j}} U_{i}\right)\right)=g\left(A_{\xi_{i}}^{*} \xi_{j}, F\left(A_{N_{j}} U_{i}\right)\right)=0 .
$$

From $(17)_{2},(24)_{4},(49)_{4}$ and the fact that $h_{a}^{s}$ is symmetric, we get

$$
h_{i}^{\ell}\left(\xi_{j}, W_{a}\right)=\epsilon_{a} h_{a}^{s}\left(\xi_{j}, V_{i}\right)=\epsilon_{a} h_{a}^{s}\left(V_{i}, \xi_{j}\right)=-\lambda_{a j}\left(V_{i}\right)=0 .
$$

Applying $\nabla_{X}$ to $(58)_{2}: h_{i}^{\ell}\left(Y, U_{j}\right)=0$ and using (25), we have

$$
\begin{aligned}
\left(\nabla_{X} h_{i}^{\ell}\right)\left(Y, U_{j}\right) & =-h_{i}^{\ell}\left(Y, F\left(A_{N_{j}} X\right)\right)- \\
& -\sum_{a=r+1}^{a} \rho_{j a}(X) h_{i}^{\ell}\left(Y, W_{a}\right)-\beta_{j} h_{i}^{\ell}(Y, F X)-\theta\left(U_{j}\right) h_{i}^{\ell}(X, Y) .
\end{aligned}
$$

Substituting this into (66) with $Z=U_{j}$ and using $(58)_{2}$, we get

$$
\begin{aligned}
& h_{i}^{\ell}\left(X, F\left(A_{N_{j}} Y\right)\right)-h_{i}^{\ell}\left(Y, F\left(A_{N_{j}} X\right)\right)+ \\
& +\sum_{a=r+1}^{n}\left\{\rho_{j a}(Y) h_{i}^{\ell}\left(X, W_{a}\right)-\rho_{j a}(X) h_{i}^{\ell}\left(Y, W_{a}\right)\right\}+ \\
& +\sum_{a=r+1}^{n}\left\{\lambda_{a i}(X) h_{a}^{s}\left(Y, U_{j}\right)-\lambda_{a i}(Y) h_{a}^{s}\left(X, U_{j}\right)\right\}+ \\
& \quad+\beta_{j}\left\{h_{i}^{\ell}(X, F Y)-h_{i}^{\ell}(Y, F X)\right\}= \\
& \quad=\frac{c}{4}\left\{u_{i}(Y) \eta_{j}(X)-u_{i}(X) \eta_{j}(Y)+2 \delta_{i j} \bar{g}(X, J Y)\right\} .
\end{aligned}
$$

Taking $Y=U_{i}$ and $X=\xi_{j}$ to this equation and using $(49)_{3,5},(58)_{2},(70)$ and (71), we have $c=0$. Consequently, $\bar{M}(c)$ is flat.

(3) As $\nabla_{X} U_{i}=0$, taking the scalar product with $U_{j}$ to (25), we get

$$
\eta_{j}\left(A_{N_{i}} X\right)=-\beta_{i} \eta_{j}(X)+\theta\left(U_{i}\right) v_{j}(X) .
$$

Substituting this equation into the left term of $(17)_{3}$, we have

$$
\theta\left(U_{i}\right) v_{j}(X)+\theta\left(U_{j}\right) v_{i}(X)=0 .
$$

Taking $X=V_{j}$ to this equation, we obtain

$$
\theta\left(U_{i}\right)=0, \quad \eta_{j}\left(A_{N_{i}} X\right)=-\beta_{i} \eta_{j}(X) .
$$


Applying $\bar{\nabla}_{X}$ to $\theta\left(U_{i}\right)=0$ and using (7) and $\nabla_{X} U_{i}=0$, we get

$$
\left(\bar{\nabla}_{X} \theta\right)\left(U_{i}\right)=-\sum_{k=1}^{r} \beta_{k} h_{k}^{\ell}\left(X, U_{i}\right)-\sum_{a=r+1}^{n} \gamma_{a} h_{a}^{s}\left(X, U_{i}\right) .
$$

Taking the scalar product with $W_{a}$ and $N_{j}$ to (25) by turns and using (16) and $(72)_{1}$, we have

$$
\rho_{i a}=0, \quad h_{i}^{*}\left(X, U_{j}\right)=0 .
$$

From $(17)_{4}$ and $(74)_{1}$, we see that

$$
\eta_{i}\left(A_{E_{a}} X\right)=-\gamma_{a} \eta_{i}(X)
$$

Applying $\nabla_{Y}$ to $(74)_{2}$ and using the fact that $\nabla_{X} U_{j}=0$, we obtain

$$
\left(\nabla_{X} h_{i}^{*}\right)\left(Y, U_{j}\right)=0 \text {. }
$$

Replacing $P Z$ by $U_{j}$ to (66) and using $(72)_{2},(73),(74)_{2},(75)$ and the last equation, we have

$$
\frac{c}{4}\left\{v_{j}(Y) \eta_{i}(X)-v_{j}(X) \eta_{i}(Y)+v_{i}(Y) \eta_{j}(X)-v_{i}(X) \eta_{j}(Y)\right\}=0 .
$$

Taking $X=\xi_{i}$ and $Y=V_{j}$ to this equation, we have $c=0$.

(4) As $\nabla_{X} V_{i}=0$, taking the scalar product with $V_{j}, W_{a}$ and $N_{j}$ to (26) by turns and using (14) and $(17)_{2}$, we obtain

$$
\begin{gathered}
h_{j}^{\ell}\left(X, \xi_{i}\right)=-\theta\left(V_{i}\right) u_{j}(X), \quad h_{a}^{s}\left(X, \xi_{i}\right)=-\theta\left(V_{i}\right) w_{a}(X), \\
h_{i}^{\ell}\left(X, U_{j}\right)=-\theta\left(V_{i}\right) \eta_{j}(X) .
\end{gathered}
$$

By using $(24)_{4},(76)_{3}$ and the fact that $h_{i}^{\ell}$ is symmetric, we see that

$$
h_{a}^{s}\left(U_{j}, V_{k}\right)=\epsilon_{a} h_{k}^{\ell}\left(U_{j}, W_{a}\right)=0 .
$$

From $(24)_{1}$ and $(76)_{3}$, we obtain $h_{i}^{*}\left(Y, V_{j}\right)=0$. Applying $\nabla_{X}$ to this equation and using the fact that $\nabla_{X} V_{j}=0$, we get

$$
\left(\nabla_{X} h_{i}^{*}\right)\left(Y, V_{j}\right)=0 \text {. }
$$

Taking $P Z=V_{j}$ to (66) and using the last two equations, we obtain 


$$
\begin{aligned}
& \sum_{j=1}^{r}\left\{h_{k}^{\ell}\left(X, V_{j}\right) \eta_{i}\left(A_{N_{k}} Y\right)-h_{k}^{\ell}\left(Y, V_{j}\right) \eta_{i}\left(A_{N_{k}} X\right)\right\}+ \\
& +\sum_{a=r+1}^{n}\left\{h_{a}^{s}\left(X, V_{j}\right) \eta_{i}\left(A_{E_{a}} Y\right)-h_{a}^{s}\left(Y, V_{j}\right) \eta_{i}\left(A_{E_{a}} X\right)\right\}- \\
& -\left(\bar{\nabla}_{X} \theta\right)\left(V_{j}\right) v_{i}(Y)+\left(\bar{\nabla}_{Y} \theta\right)\left(V_{j}\right) v_{i}(X)= \\
& \quad=\frac{c}{4}\left\{u_{j}(Y) \eta_{i}(X)-u_{j}(X) \eta_{i}(Y)+2 \delta_{i j} \bar{g}(X, J Y)\right\}
\end{aligned}
$$

Taking $X=\xi_{i}$ and $Y=U_{j}$ and using (76) and (77), we get $c=0$.

Theorem 4. Let $M$ be a generic lightlike submanifold of an indefinite complex space form $\bar{M}(c)$ with a semi-symmetric non-metric connection. If $W_{a}$ is parallel with respect to $\nabla$ and $\sum_{k=1}^{r} \beta_{k} h_{a}^{s}\left(W_{a}, V_{k}\right) \neq 0$, then $r=1$ and $c=0$.

Proof. As $\nabla_{X} W_{a}=0$, taking the scalar product with $W_{b}$ to (27), we get

$$
\mu_{a b}(X)=-\theta\left(W_{a}\right) w_{b}(X) .
$$

Substituting this equation into the left term of $(17)_{5}$, we have

$$
\epsilon_{b} \theta\left(W_{a}\right) w_{b}(X)+\epsilon_{a} \theta\left(W_{b}\right) w_{a}(X)=0 .
$$

Replacing $X$ by $W_{b}$ to the last equation, we obtain

$$
\theta\left(W_{a}\right)=0, \quad \mu_{a b}=0
$$

Applying $\bar{\nabla}_{X}$ to $\theta\left(W_{a}\right)=0$ and using (7) and $\nabla_{X} W_{a}=0$, we get

$$
\left(\bar{\nabla}_{X} \theta\right)\left(W_{a}\right)=-\sum_{i=1}^{r} \beta_{i} h_{i}^{\ell}\left(X, W_{a}\right)-\sum_{a=r+1}^{n} \gamma_{b} h_{b}^{s}\left(X, W_{a}\right) .
$$

Taking the scalar product with $U_{i}, V_{i}$ and $N_{i}$ to (27) by turns and using (15), (17) 4 and (78) 1 , we have

$$
\eta_{i}\left(A_{E_{a}} X\right)=-\gamma_{a} \eta_{i}(X) \text {, i. e., } \rho_{\mathrm{ia}}=0, \quad \lambda_{\mathrm{ai}}=0, \quad \mathrm{~h}_{\mathrm{a}}^{\mathrm{s}}\left(\mathrm{X}, \mathrm{U}_{\mathrm{i}}\right)=0 .
$$

As $\lambda_{a i}=0$, from $(17)_{2}$, we obtain

$$
h_{a}^{s}\left(X, \xi_{i}\right)=0
$$


From $(24)_{2},(78)_{1}$ and $(80)_{3}$, we obtain $h_{i}^{*}\left(X, W_{a}\right)=0$. Applying $\nabla_{Y}$ to this equation and using the fact that $\nabla_{X} W_{a}=0$, we get

$$
\left(\nabla_{X} h_{i}^{*}\right)\left(Y, W_{a}\right)=0 .
$$

Replacing $P Z$ by $W_{a}$ to (67) and using $(24)_{4},(79),(80)_{1}$ and the last two equations, we have

$$
\begin{aligned}
\sum_{k=1}^{r} h_{a}^{s}\left(X, V_{k}\right)\left\{\eta_{i}\left(A_{N_{k}} Y\right)+\beta_{k} \eta_{i}(Y)\right\}- & \sum_{k=1}^{r} h_{a}^{s}\left(Y, V_{k}\right)\left\{\eta_{i}\left(A_{N_{k}} X\right)+\beta_{k} \eta_{i}(X)\right\}= \\
& =\frac{c}{4}\left\{w_{a}(Y) \eta_{i}(X)-w_{a}(X) \eta_{i}(Y)\right\} .
\end{aligned}
$$

Taking $X=\xi_{i}$ and $Y=W_{a}$ to this and using (81), we have

$$
\sum_{k=1}^{r} h_{a}^{s}\left(W_{a}, V_{k}\right)\left\{\eta_{i}\left(A_{N_{k}} \xi_{i}\right)+\beta_{k}\right\}=-\frac{c}{4} .
$$

Comparing the co-screen components of (62) and (63), we obtain

$$
\begin{aligned}
& \left(\nabla_{X} h_{a}^{s}\right)(Y, Z)-\left(\nabla_{Y} h_{a}^{s}\right)(X, Z)+ \\
& \quad+\sum_{i=1}^{r}\left\{\rho_{i a}(X) h_{i}^{\ell}(Y, Z)-\rho_{i a}(Y) h_{i}^{\ell}(X, Z)\right\}+ \\
& \quad+\sum_{b=r+1}^{n}\left\{\mu_{b a}(X) h_{b}^{s}(Y, Z)-\mu_{b a}(Y) h_{b}^{s}(X, Z)\right\}- \\
& \quad-\theta(X) h_{a}^{s}(Y, Z)+\theta(Y) h_{a}^{s}(X, Z) \\
& \quad=\frac{c}{4}\left\{w_{a}(X) \bar{g}(J Y, Z)-w_{a}(Y) \bar{g}(J X, Z)+2 w_{a}(Z) \bar{g}(X, J Y)\right\}
\end{aligned}
$$

As $\lambda_{a i}=\mu_{a b}=\theta\left(W_{a}\right)=0$ and $F W_{b}=0$, from (27), we have

$$
F\left(A_{E_{a}} X\right)=-\gamma_{a} F X, \quad F\left(A_{E_{a}} W_{b}\right)=0 .
$$

Applying $\nabla_{X}$ to $h_{a}^{s}\left(Y, U_{i}\right)=0$ and using (25) and $(80)_{3}$, we get

$$
\left(\nabla_{X} h_{a}^{s}\right)\left(Y, U_{i}\right)=-h_{a}^{s}\left(Y, F\left(A_{N_{i}} X\right)\right)-\beta_{i} h_{a}^{s}(F X, Y)-\theta\left(U_{i}\right) h_{a}^{s}(X, Y)
$$

due to $\rho_{i a}=0$. Substituting this into (83) with $Z=U_{i}$ and using the fact that $\rho_{i a}=\mu_{a b}=0$, we have

$$
h_{a}^{s}\left(X, F\left(A_{N_{i}} Y\right)\right)-h_{a}^{s}\left(Y, F\left(A_{N_{i}} X\right)\right)+\beta_{i}\left\{h_{a}^{s}(X, F Y)-h_{a}^{s}(F X, Y)\right\}=
$$




$$
=\frac{c}{4}\left\{w_{a}(Y) \eta_{i}(X)-w_{a}(X) \eta_{i}(Y)\right\} .
$$

Taking $X=\xi_{i}$ and $Y=W_{a}$ to this equation and using (81), we get

$$
h_{a}^{s}\left(W_{a}, F\left(A_{N_{i}} \xi_{i}\right)\right)-\beta_{i} h_{a}^{s}\left(V_{i}, W_{a}\right)=-\frac{c}{4} .
$$

From $(5)_{2},(15),(17)_{3,4},(22),(84)_{2}$ and the fact: $\rho_{i a}=0$, we have

$$
\begin{aligned}
h_{a}^{s}\left(W_{a}, F\left(A_{N_{i}} \xi_{i}\right)\right)=-\epsilon_{a} g\left(A_{N_{i}} \xi_{i}, F\right. & \left.\left(A_{E_{a}} W_{a}\right)\right)- \\
-\sum_{k=1}^{r} h_{a}^{s}\left(W_{a}, V_{k}\right) \eta_{k}\left(A_{N_{i}} \xi_{i}\right) & =-\sum_{k=1}^{r} h_{a}^{s}\left(W_{a}, V_{k}\right) \eta_{k}\left(A_{N_{i}} \xi_{i}\right)= \\
& =\sum_{k=1}^{r} h_{a}^{s}\left(W_{a}, V_{k}\right)\left\{\eta_{i}\left(A_{N_{k}} \xi_{i}\right)+2 \beta_{k}\right\} .
\end{aligned}
$$

From the last two equations, we see that

$$
\sum_{k=1}^{r} h_{a}^{s}\left(W_{a}, V_{k}\right)\left\{\eta_{i}\left(A_{N_{k}} \xi_{i}\right)+2 \beta_{k}\right\}-\beta_{i} h_{a}^{s}\left(W_{a}, V_{i}\right)=-\frac{c}{4} .
$$

Comparing this equation with (82), we obtain

$$
\sum_{k=1}^{r} \beta_{k} h_{a}^{s}\left(W_{a}, V_{k}\right)=\beta_{i} h_{a}^{s}\left(W_{a}, V_{i}\right), \quad \forall i
$$

It follow that

$$
(r-1) \sum_{k=1}^{r} \beta_{k} h_{a}^{s}\left(W_{a}, V_{k}\right)=0 .
$$

Assume that $\sum_{k=1}^{r} \beta_{k} h_{a}^{s}\left(W_{a}, V_{k}\right) \neq 0$. Then $r=1$ and $i=j=k=1$. Thus, from $(17)_{3}$, we see that

$$
\eta_{i}\left(A_{N_{1}} X\right)=-\beta_{1} \eta_{1}(X) .
$$

From this result and (82), we obtain $c=0$.

Acknowledgment. In this paper, we studied the geometry of generic lightlike submanifolds of an indefinite Kaehler manifold with a semi-symmetric non-metric connection. But the geometry of generic lightlike submanifolds and several CR-type lightlike submanifolds of an indefinite Kaehler manifold with a quarter-symmetric non-metric connection are still open problems. We hope that the publication of this paper will help in solving the above more general cases. 


\section{References}

[1] Ageshe N.S., Chafle M.R. A semi-symmetric non-metric connection on a Riemannian manifold. Indian J. Pure Appl. Math., 1992, vol. 23, no. 6, pp. $399-409$.

[2] Duggal K. L., Bejancu A. Lightlike Submanifolds of Semi-Riemannian Manifolds and Applications. Kluwer Acad. Publishers, Dordrecht, 1996.

[3] Duggal K. L., Jin D.H. Generic lightlike submanifolds of an indefinite Sasakian manifold. Int. Elec. J. Geo., 2012, vol. 5, no. 1, pp. 108-119.

[4] Jin D.H. Indefinite generalized Sasakian space form admitting a generic lightlike submanifold. Bull. Korean Math. Soc., 2014, vol. 51, no. 6, pp. 1711-1726. DOI: https://doi.org/10.4134/BKMS .2014.51.6.1711.

[5] Jin D.H. Generic lightlike submanifolds of an indefinite trans-Sasakian manifold of a quasi-constant curvature. Appl. Math. Sci., 2015, vol. 9, no. 60, pp. 2985 - 2997. DOI: http://dx.doi.org/10.12988/ams. 2015. 52165.

[6] Jin D. H. Special lightlike hypersurfaces of indefinite Kaehler manifolds. Filomat, 2016, vol. 30, no. 7, pp. 1919-1930.

[7] Jin D. H. Lightlike hypersurface of an indefinite Kaehler manifold with a semi-symmetric non-metric connection. J. Korean Math. Soc., 2017, vol. 54, no. 1, pp. 101-115.

[8] Jin D.H., Lee J.W. Generic lightlike submanifolds of an indefinite cosymplectic manifold. Math. Probl. in Engin., 2011, Art ID 610986, pp. 1-16.

[9] Kupeli D. N. Singular Semi-Riemannian Geometry, Kluwer Academic, 1996, 366 p.

[10] De Rham G. Sur la réductibilité d'un espace de Riemannian. Comm. Math. Helv., 1952, vol. 26, pp. $328-344$.

Received April 12, 2018.

In revised form, July 27, 2018.

Accepted September 27, 2018.

Published online October 11, 2018.

Department of Mathematics

Dongguk University

Gyeongju 780-714, Republic of Korea

E-mail: jindh@dongguk.ac.kr 